\title{
The potential of thromboelastometry for the diagnosis and treatment of coagulopathy in patients undergoing craniotomy due to isolated traumatic brain injury (TBI)
}

\author{
M. Rimaitis ${ }^{1}$, D. Bilskiene ${ }^{1}$, T. Tamosuitis ${ }^{2}$, R. Vilcinis ${ }^{2}$, A. Macas ${ }^{1}$ \\ ${ }^{1}$ Lithuanian University of Health Sciences, Clinic of Anesthesiology \\ 2Lithuanian University of Health Sciences, Clinic of Neurosurgery
}

Background: Craniotomy to treat traumatic brain injury is a major neurosurgical intervention associated with significant bleeding. Adequate assessment of coagulation status is crucial as coagulopathy (CGP) in patients with TBI is prevalent. Rotational thromboelastmetry (ROTEM) is a coagulation assay proven to be useful in trauma and major surgery.

Aim of the Study: To compare preoperative standard coagulation test (SCT) and ROTEM results and evaluate their efficacy to predict intraoperative blood loss in neurosurgical isolated TBI patients.

Materials and Methods: We performed a prospective, observational pilot study in a cohort of 32 adult patients requiring urgent craniotomy. Isolated severe TBI was defined as Abbreviated Injury Scale for head $\left(\right.$ AIS $\left._{\text {head }}\right)>3$ and $\mathrm{AIS}_{\text {extracranial }}<3$. Blood was collected preoperatively and analyzed with SCT (prothrombin time index [PTI], partial thromboplastin time [APTT], platelet count [PLT], fibrinogen concentration [Fib.]), and ROTEM assays (EXTEM, INTEM, FIBTEM). CGP was defined as any abnormality on ROTEM and/or PTI $<70 \%$, APTT $>38 \mathrm{~s}$, Fib. $<1.5 \mathrm{~g} / \mathrm{L}, \mathrm{PLT}<100 * 10^{\%} / \mathrm{L}$. Significant blood loss was considered $>300 \mathrm{~mL}$. Study patients were allocated into low blood loss (LBL) and significant blood loss (SBL) groups, and comparative analysis was performed.

Results and Discussion: Overall incidence of CGP was $40.6 \%(\mathrm{~N}=13), 31.3 \%(\mathrm{~N}=10)$, and $53.1 \%(\mathrm{~N}=17)$ according to SCT; ROTEM; and SCT/ROTEM, respectively. Median blood loss in study cohort was 350 (Q1-Q3, 300-500)ml. Significant ROTEM parameter differences (CT, CFT, alpha angle, A10, MCF) between significant and low blood loss groups were detected, whereas according to SCT, only Fib. differed (table 1). Despite Fib. $>1.5 \mathrm{~g} / \mathrm{L}$ in all cases, there were 4 patients (12\%) with impaired clot firmness according to ROTEM findings (figure 1). Significant relationship between CGP in ROTEM as well as in ROTEM/SCT, and SBL was detected. Sensitivity and specificity of CGP in ROTEM to predict SBL was $50 \%$ and $87.5 \%$. CGP in SCT/ROTEM predicted SBL with $68.8 \%$ sensitivity and $75 \%$ specificity.

Conclusion: ROTEM may aid in predicting blood loss, and can be superior in characterizing and optimizing coagulation, especially if SCT values are marginal. Lower target for fibrinogen $(1.5 \mathrm{~g} / \mathrm{L})$ in TBI may be too low.

\begin{tabular}{|c|c|c|c|}
\hline & LBL group, $n=16$ & SBL group, $\mathrm{n}=16$ & \multirow[b]{2}{*}{ p-value } \\
\hline & \multicolumn{2}{|c|}{ Expressed as median (Q1-Q3) } & \\
\hline Admission GCS & $10(5.5-13.5)$ & $5.5(4-10)$ & $0.034 *$ \\
\hline \multicolumn{4}{|c|}{ Standard laboratory tests } \\
\hline Hemoglobin, g/L & $127.5(120.25-142.75)$ & $135.5(119.5-145.5)$ & 0.692 \\
\hline Platelet count, $* 10^{9} / \mathrm{L}$ & $190(148.25-257)$ & $183(103-263.75)$ & 0.749 \\
\hline PTI, \% & $99.5(83.5-117.75)$ & $92.5(79-109.25)$ & 0.611 \\
\hline INR & $1.01(0.96-1.08)$ & $1.03(0.96-1.11)$ & 0.650 \\
\hline APTT, s & $31.6(28.4-34.63)$ & $31.25(29.2-32.9)$ & 0.763 \\
\hline Fibrinogen, g/L & $3.83(3-4.2)$ & $2.32(1.92-2.86)$ & $0.006 *$ \\
\hline \multicolumn{4}{|c|}{ Thromboelastometry } \\
\hline INTEM CT, $\mathrm{s}$ & $156(138.75-170.5)$ & $155(135.25-198.75)$ & 0.763 \\
\hline INTEM CFT, $\mathrm{s}$ & $78.5(64.25-99)$ & $96.5(80-141.75)$ & 0.055 \\
\hline INTEM alpha angle & 74.5(73-77.75) & $72(66-74)$ & $0.041 *$ \\
\hline INTEM A10, mm & $55(47.5-60)$ & $48(40.5-51.75)$ & $0.010 *$ \\
\hline INTEM MCF, mm & $62.5(56.5-66.75)$ & $56.5(50.25-61.5)$ & $0.010 *$ \\
\hline INTEM ML, \% & $4(2.25-7)$ & $3.5(2-6.75)$ & 0.582 \\
\hline EXTEM CT, s & $56(50.75-59.5)$ & $69.5(53.25-79)$ & $0.033^{*}$ \\
\hline EXTEM CFT, $\mathrm{s}$ & $79(59-107.25)$ & $104.5(78.5-152.25)$ & $0.016 *$ \\
\hline EXTEM alpha angle & $77.5(72-78)$ & $70.5(61.25-76)$ & $0.010 *$ \\
\hline EXTEM A10, mm & $55(49.5-62.5)$ & $47.5(40.25-52)$ & $0.004 *$ \\
\hline EXTEM MCF, mm & $63(57.25-67.75)$ & $54.5(50.5-60.75)$ & $0.004^{*}$ \\
\hline EXTEM ML, \% & $4.5(3.25-7.75)$ & $5(2.25-6)$ & 0.676 \\
\hline FIBTEM CT, $\mathrm{s}$ & $53(47.25-72)$ & $63(47.5-83.75)$ & 0.534 \\
\hline FIBTEM $\mathrm{A} 10, \mathrm{~mm}$ & $20(15.25-24)$ & $13(8-19.75)$ & $0.008 *$ \\
\hline FIBTEM MCF, mm & $22.5(17-29.25)$ & $15.5(8.25-22)$ & $0.006 *$ \\
\hline FIBTEM ML, \% & $0(0-3)$ & $0.5(0-3)$ & 0.225 \\
\hline \multicolumn{4}{|c|}{ Coagulopathy (\%) } \\
\hline CGP acc. to SCT & $5(31.3 \%)$ & $8(50 \%)$ & 0.28 \\
\hline CGP acc. to ROTEM & $2(12.5 \%)$ & $8(50 \%)$ & $0.022 *$ \\
\hline $\begin{array}{l}\text { CGP acc. to ROTEM } \\
\text { and/or SCT }\end{array}$ & $5(31.3 \%)$ & $12(75 \%)$ & $0.013 *$ \\
\hline
\end{tabular}

*Significant differences between groups, $p<0.05$.

GCS - Glasgow coma score, CT - clotting time, CFT - clot formation time, A10 - clot amplitude after 10 minutes, MCF - maximum clot firmness, ML - maximum lysis.

Non-parametric Mann-Whitney $U$ and Pearson Chi-square tests were used as applicable.

Figure 1. ROTEM (EXTEM and FIBTEM) tracings reflecting low clot firmness due to insufficient fibrinogen contribution.

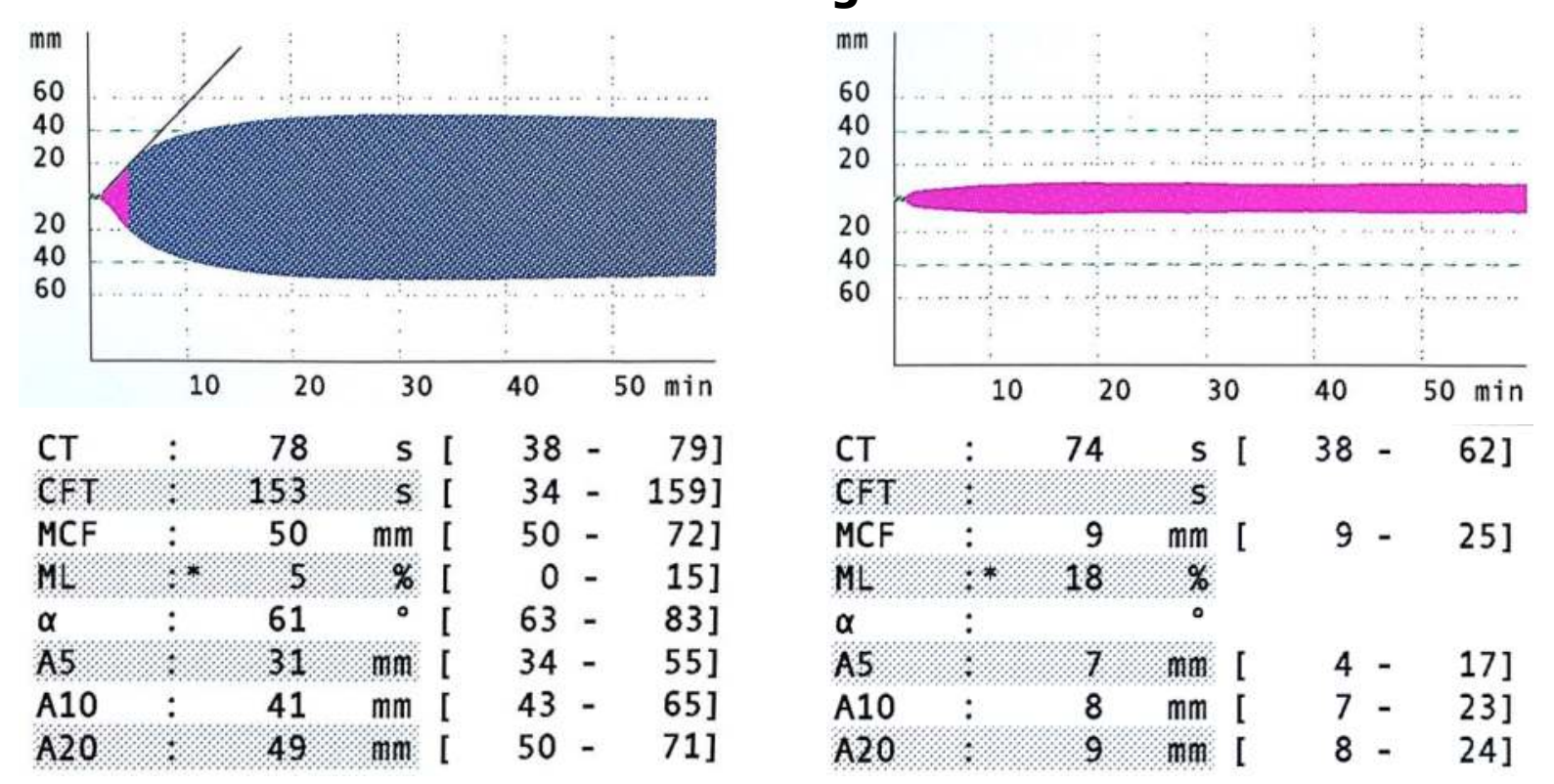

